\title{
Analysis of Meituan Community Group Purchase Mode
}

\author{
Yucong $\mathrm{Li}^{1,}{ }^{\dagger}$, Xiaoxiao $\mathrm{Mei}^{2,{ }^{*},{ }^{\dagger}}$ \\ ${ }^{1}$ School of arts and social sciences, the University of Sydney, Sydney, Australia \\ ${ }^{2}$ School of Information Mechanical and Electrical Engineering, Shanghai Normal University, \\ Shanghai, China \\ ${ }^{*}$ Corresponding author: 1000467755@smail.shnu.edu.cn \\ These authors contributed equally.
}

\begin{abstract}
Community group buying is a new retail model that relies on the community and the social relationship of the head of the group to realize the circulation of fresh goods. Starting from the development and current situation of community group buying model, we analyze the business model, competitiveness and other aspects through literature research method and case study method, and mainly use SWOT analysis to analyze the strengths, weaknesses, opportunities and threats of Meituan youxuan at multiple levels. Compared with other companies of the same type, Meituan youxuan has advantages in talent strategy, core technology, and operation, and disadvantages in supply management and individual business competitiveness. However, due to the longer-term impact of the epidemic gradually changing the way consumers consume, this will become an opportunity for the industry. At the same time the tightening of relevant national policies emphasizes the need to bring the utility and benefits of users, suppliers and platforms into dynamic balance to further ensure the long-term optimistic development of the business. Meituan youxuan needs to build core competencies and form an efficient supply chain and logistics system for the industry barriers of fresh community group buying.
\end{abstract}

Keywords: Meituan youxuan, SWOT, Community group buying, Meituan, Fresh produce electronic business.

\section{Introduction}

Since the pandemic outbreak in 2019, community bulk order became a new method. Under the current background about the Internet continues to develop, community bulk order aggression and importance. This model has a few innovations, it does not only directly affect the people's consumption patterns and consumption habits, but it also induces various contradictions and problems, when the supervision of community bulk order is limited, it may directly affect the development of the Internet economy. Meituan designated the community bulk order as a "Top strategic project", it became a new pioneer in addition to the two main businesses of food delivery, in-store and hotel. The community bulk order war ended the initial stage of capital competition. The initial investment has led to a significant loss of each platform, resources are intended to be pro-customer when the tightening of supervision. Meituan Optimum has good market conditions and business models. At this stage, strengthening the supply chain can achieve a high-efficiency "three-in-one", and it has a good development pattern.

\section{Literature Review}

Strategic management in a broad sense refers to the use of strategy to manage the entire enterprise. Chandler, a famous American management scientist, analyzed the relationship among environment, strategy and organizational structure in his book Strategy and Structure. He believed that business strategy should adapt to the environment - to meet the needs of the market, and that the organizational structure must adapt to the strategy and change with it. Later, two schools of study on strategic structure came into being: "Design school" and "planning school" (Geng, 1999).

The planning school is mainly represented by Ansoff et al. (1976). Ansoff et al. (1976) studied the basic principles, theories and procedures of enterprise development in Corporate Strategy published 
in 1965, and preliminarily formed the theoretical framework of enterprise strategic management research (Feng, 2008). In addition, Ansoff et al. (1976) put forward the concept of "enterprise strategic management". In his opinion, strategic management of an enterprise refers to a series of operation and management businesses formed by combining daily business decisions with long-term planning decisions. The representative figure of the design school is the famous professor Andrews of Harvard Business School and his colleagues. The basic model proposed by them is to divide the strategic structure into two parts: formulation and implementation, and SWOT analysis is used in the process of making (Zuo \& Xu, 2001). To sum up, the essence of traditional strategic management is the process of an organization's adaptation to its environment, as well as the process of internal structure changes caused by it. However, it ignores the analysis of the industry and competitive environment in which the enterprise is located, making the enterprise in a state of passive adaptation to the external environment (Bai \& Bai, 2005). With the development of enterprise strategy theory and enterprise management practice, the research focus of enterprise strategy theory is gradually transferred to enterprise competition. In the 1980s, Michael Porter of Harvard University put forward the theory of competitive positioning. Porter believed that the core of enterprise strategy is to gain competitive advantage. The competitive strategy is based on the selection of attractive industries with high potential profits, and then to determine their competitive position in the selected industries. The theory of competitive positioning shifts the focus of enterprise strategy analysis from enterprise to industry, emphasizes the importance of external environment, and provides extremely useful analytical tools such as industry attraction matrix and value chain analysis for the process of strategy selection (Ren, 2016).

\section{Domestic Research Literature}

The theoretical research on strategic management in China started late. After the reform and opening up, domestic scholars only started to conduct research, but the development was relatively fast and deep, mainly including the theory of core competitiveness, the theory of enterprise diversification, the theory of enterprise development strategy based on human orientation and the theory of system based on complexity science, the theory of business ecosystem, etc. (Zhang \& Zhou, 2004). Guo and Zhou (2004) proposed that the growth strategy of enterprises should follow the principle of relevance and the principle of attractiveness, i.e., choose industries that are related to the original business and are attractive. In 2010, Tong (2011) proposed that strengthening corporate culture can cultivate market awareness, promote system reform, and advance brand building. In 2019, Liu (2020) proposed that companies should improve the strategic management awareness of all employees and use professional methods and theories to guide work time. In 2020, Qu (2020) proposed that the company's growth strategy should be based on the theory of complexity science and the theory of business ecosystem. Qu (2020) proposed that to obtain strategic and competitive advantages in the context of internationalization, we should promote both government regulation and guidance, and enterprises' independent innovation and quality improvement.

In the theoretical neighborhood of e-commerce retailing, "new retail" and other types of strategic management, Hong and Li (2015), after analyzing the structural adjustment of China's retail industry, propose that: due to the impact of e-commerce, the traditional retail industry should recognize that the era of rapid economic and consumer growth is over, and in order to avoid a massive tide of store closures, in order to avoid massive store closures, they can expand and sink their sales channels to third and fourth-tier cities. At the same time, mergers and reorganization and multi-format retailers will rise, and industry leaders will explore the full experience of business model. Through their research, Du and Jiang (2017) concluded that the typical characteristics of the "new retail" are ecological, borderless, intelligent, and experiential. Ecology" is the most basic feature, which means that the coordinated integration of online and offline requires a complete and efficient ecosystem to guarantee: "Borderlessness" means breaking all kinds of barriers in retail channels, and integrating all platforms and services in an "omni-channel" way. "Smart" means that "new retail" must rely on 
the advancement and widespread use of high technology in order to be successfully realized; "Experiential" means that the "new retail" will be able to integrate all platforms and resources in an "omni-channel" manner. "Experiential" is the mainstream business method widely used by "new retail" companies, and the good or bad experience directly determines whether consumer behavior occurs or not.

Therefore, this article takes Meituan's subordinate Meituan Optimum as the research object, conduct a more comprehensive analysis from the aspects of business model and competitiveness with literature research method, case study method and etc. This article focuses on the community bulk order model in the post-epidemic era and the strategy management of Meituan Optimal, then put some targeted suggestions on its future development trend. Establish core competitiveness to create internet food industry's barrier under the community bulk order model, and provide some valuable and meaningful experiences for similar companies or other companies which want to go to a new business frontier.

\section{Data and Method}

\subsection{Data}

Meituan is a pioneer in the new big-tech service industry and a tycoon in Chinese new internet companies. Involved in store business, food delivery, travel, hotel and other fields, because of its diverse sources of customers, it provides a solid customer foundation for Meituan Optimal. Founded in March 2010, Meituan is a platform company that provides food, housing, transportation and other life services with both consumption and technology attributes. Meituan adopts the Daily Deals model which is pushing selected content every day. By doing this it realized the online commercialization of offline catering, entertainment and other business information. Also, it uses IT, CMS and other systems to improve compliance efficiency and reduce costs. Community group is the strategic point of Meituan. See the certainty in the uncertainty. After the initial running of the business model, the supervision of the industry competition gradually return to normal. Community group is an efficiency improvement from the supply chain to the performance chain. Commodity preference itself attracts users, while the mid-term core barrier lies in the reduction of back-end performance cost. When community group-buying model is completed, it can achieve $0-5 \%$ profit margins.

\subsection{Method}

This paper adopts SWOT analysis method to analyze the current business model of Meituan youxuan.

SWOT is a matrix thinking framework that analyzes the environment of an enterprise and its business from the perspectives of strengths, weaknesses, opportunities and threats through the internal and external competitive environment and the situation under competitive conditions. Then with the thought of systematic analysis, all kinds of factors are matched to be analyzed, from which a series of corresponding conclusions are drawn, and the conclusions usually lead to certain decision. Due to the limited resources and strength of the enterprise, it is very important to establish the comparative advantage of the enterprise.

Advantage-Opportunity (SO) strategy is a kind of strategy that gives full play to the internal advantages of an enterprise to take advantage of external opportunities. Which means taking advantage of your internal strengths to seize and make full use of opportunities provided by changes in the external environment. Enterprises usually need to go through WOIST or WT strategies to be able to adopt SO strategies.

The goal of the Weak-Opportunity (WO) strategy is to compensate for internal weaknesses by taking advantage of external opportunities. In general, there are internal weaknesses that prevent an enterprise from taking advantage of external opportunities that may exist

Advantage-Threat (ST) strategy is to use the advantages of the enterprise to avoid or reduce the impact of external threats. Of course, the threat may come from the cross-border attack of a third party, or the potential impact of new products, new materials and new technologies on existing 
enterprises. Enterprises should make good use of the current advantages, do a good job of coping strategies, so as to have a breakthrough.

Disadvantage-Threat (WT) strategy is a defensive strategy aiming at reducing internal disadvantages while avoiding external environmental threats. Enterprises at this stage are very precarious, any carelessness may lead to mergers and acquisitions, contraction, bankruptcy or liquidation. Therefore, enterprises at this stage must fighting with a readiness to die. They must get rid of the unnecessary decisively, like cutting redundant people, getting rid of unprofitable businesses, closing loss-making stores, etc. And only retain the strongest point, recuperate, rectify management and business, and wait for the opportunity (Shen, 2009).

\section{Results and Discussion}

\subsection{Strength:}

\subsubsection{Branding and Technical Support}

With super application support, it has a natural spreading effect in consumers' mind and can bring a lot of streams. With years of construction in the retail field, Meituan now has a very sophisticated business center and data center, and has the technical advantages in efficiently responding to business changes and intelligent customer management.

\subsubsection{Talent Strategies}

As the winner of the "Thousand Group War", Meituan has a strong ground push team, "internal transfer + high salary recruitment + local business foundation + complete job training" to seize highquality resources quickly, from the end of the supply chain to complete the user precipitation. At present, the team members of Meituan youxuan Business Division have grown to more than 3, 000, including more than 1, 000 ground pushers, and the company has set a target of 60,000 new recruits in early 2021 to reserve abundant ground pushing talents for community group purchase business.

\subsubsection{Industry-Leading Offline Operation Capability}

The offline operation capability of Meituan is obviously reflected in the takeaway war. The community group purchase business is essentially an offline retail business, which requires the platform to manage and control the whole supply chain from "supplier - central warehouse - grid warehouse - head". According to research, Meituan is very strict in controlling all aspects of community group purchase, and each aspect has corresponding operation and Business Developmentpersonnel dedicated to the responsibility and clear rewards and punishments.

\subsubsection{Supply Demand Positive Feedback}

Meituan relies on strong merchant management capabilities and has accumulated a very large number of partners, so relatively can choose the source of goods will be more high-quality and lowcost. On the one hand, Meituan will make consumers more trust Meituan to bring more orders, on the other hand, it can also reduce costs to improve gross margins.

\subsubsection{Formation of "Beauty Group" Ecological Effect}

Meituan buy food focus on the north, Guangzhou and Shenzhen and other super first-tier cities; Meituan flash purchase under the vegetable Daquan is focused on Wuhan, Chengdu, Nanjing and other quasi first-tier or second and third-tier cities; Meituan youxuan is aimed at the sinking market, the three lines together, Meituan want to form a front warehouse, vegetable market operations, community group purchase and other relatively complete multi-state layout.

\subsubsection{Clear and Unshakable Long-Term Strategic Positioning}

Meituan has positioned its community group purchase business in a long-term layout of three to five years, so it is not limited to immediate interests and does not worry about short-term losses. The company increase its strategic investment in new businesses in the first quarter of 2021, expanding 
its losses to 8 billion yuan in a single quarter, with community group losses accounting for more than $70 \%$ of the total, making it the largest area of investment. The entrance of Meituan youxuan is located in the most prominent place in Meituan application, occupying almost 1/2 of Meituan home page, Meituan application provides excellent user traffic conversion basis for Meituan youxuan.

\subsection{Weakness}

\subsubsection{Logistics System Under Construction}

Compared to other companies, Meituan started late in the warehousing segment, especially in the construction of cross-city transportation, origin sourcing and other capabilities to lag behind the head of the community group buying rivals.

\subsubsection{Supply Management}

Supply chain has a strong bullwhip effect, one is to prevent shortages of goods generally from the former warehouse, large warehouse, to regional warehouse each layer will be stocked, which will cause a lot of unnecessary waste. Secondly, the slow feedback of information, such as at raining in a certain area demand surges, feedback to the source will take a lot of time. Supply problems need to be solved in an information-based way, so a lot of preliminary data accumulation is needed.

\subsubsection{Weak Competitiveness of Individual Business Capabilities}

Meituan has always been a trading platform, and has not had particularly successful experience in community operations. The model has not been finalized. Due to the lack of innovative community group buying model is still undefined, there may be certain risks.

\subsection{Opportunity}

\subsubsection{The Epidemic Affected Consumption Patterns}

The fresh produce e-commerce and community group buying models experienced explosive activity and growth in the general environment of the epidemic. During the epidemic, many places implemented travel controls for residents, and consumers chose e-commerce to purchase all fresh fruits and vegetables and other necessities in a one-stop shop. In the post-epidemic era, the next-day delivery feature of community group purchase meets the demand for reduced exposure and protection, while consumers are more conscious of life and the concept of health maintenance will maintain everyone's sense of cooking and using quality ingredients. A higher demand for community group buying model.

\subsubsection{Technology for Model Development}

Community group buying is the primary solution to consumer demand for fresh products shopping, fresh products have the characteristics of easy loss, low shelf life, and have immediate demand, these can be further empowered by technology. For example, in the era of big data, fresh produce ecommerce can use user consumption data, community user portraits to optimize product selection and accurately predict user demand in order to reduce losses and improve inventory turnover. Cold chain logistics is a key link in the value chain of fresh produce e-commerce, and is a difficult link to break through. China's cold chain technology is being developed and upgraded with the progress of logistics technology - in the future, as the Internet of Things, block chain, RFIF, intelligent temperature control and other cutting-edge technologies are further promoted and applied in cold chain logistics, cold chain traceability and full monitoring technologies are gradually formed, the quality and safety of fresh products in the cold chain transportation process will be The quality and safety of fresh products during cold chain transportation will be more effectively ensured, and fresh food e-commerce companies will be able to further reduce the cost of loss. 


\subsection{Threat}

\subsubsection{Fierce Competition in the Same Industry}

Meituan youxuan as a latecomer, in the face of the already more mature model is bound to face a lot of competition, such as the head of management, price, marketing and other levels. For example: Pinduoduo Inc's Duo Duo buy food, its positioning for the sinking market to form a natural advantage, making user awareness and user stickiness stronger. The box market is also a relatively strong competitor. First of all, it is backed by Ali and has sufficient resources in all aspects. Secondly, the infrastructure is relatively perfect, it has complete cold chain facilities and strong delivery capacity, which is ahead of other competitors in terms of fulfillment time.

\subsubsection{Infrastructure Construction}

The construction of basic warehousing and distribution requires a long time and capital investment, the basic ability to keep up may become a bottleneck for business expansion.

\section{Conclusion}

To be brief, the community bulk order war ended the initial stage of capital competition. The initial capital investment led to significant losses for each platform. When the tightening of supervision, resources have shown a friendly signal to costumers. With its good market conditions and business model, Meituan has won the community bulk order. Based on its technological advantages and sufficient local talents, Meituan insists on setting the development of community group buying as an unshakable long-term strategy. Meanwhile, based on the SWOT model, we combined with the current market conditions and proposed that Meituan Optimum at this stage which can achieve a higher efficiency "three in one" by strengthening the supply chain, which has a good development form.

Community bulk order solves the last-mile delivery problem, it is a great reforming in the retail industry. As the team work effect rises, the market space is profitable in the profitable. The offline retail business will not be a dominant phenomenon, community bulk order has obvious regional characteristics, therefore, it is particularly important to build core competitiveness and industry barriers. Competing with the construction of infrastructure such as the supply chain and cold chain of various platforms in this stage, after experience a round of inspections in the summer of next year, you can enter a new round of competition for SKU selection and improvement of group efficiency. In the end, an efficient supply chain and logistics system will become an industry barrier for community bulk order industry.

\section{Conflict of Interest}

The authors declare no conflict of interest.

\section{Author Contributions}

$\mathrm{AB}$ conducted the research; $\mathrm{CD}$ analyzed the data; $\mathrm{AB}$ wrote the paper; all authors had approved the final version.

\section{References}

[1] Ansoff, H. I., Declerck, R. P., \& Hayes, R. L. (1976). From strategic planning to strategic management [Paper presentation]. International Conference on Strategic Management 1973, Nashville, Tenn.

[2] Bai, X. P., \& Bai, F. R. (2005). The evolutionary track of strategic management theory. Enterprise Reform and Management, (4), 18-19.

[3] Du, R. Y., \& Jiang, K. (2017). New retail: Connotation, development motivation and key issues. Price Theory and Practice, (2), 139-141. doi: 10.19851/j.cnki.cn11-1010/f.2017.02.038. 
[4] Feng, X. (2008). The development course and new trend of the theory of enterprise strategic management. Scientific and Technological Information Development and Economy, (10), 169-171.

[5] Geng, H. (1999). The evolution and new development of the theory of corporate strategic management. Foreign Economics and Management, (6), 14-16. https://doi.org/10.16538/j.cnki.fem.1999.06.003

[6] Guo, J. R., \& Zhou, S. D. (2004). Discussion on the growth strategy of small and medium-sized enterprises-A case study of the growth of Jiangsu Tongrun Group. Nankai Management Review, (2), 106112.

[7] Hong, T., \& Li, G. Y. (2015). Analysis on the structural adjustment of China's retail industry-2014 China's retail industry closure report. China's Circulation Economy, (3), 1-9. doi: 10.14089/j.cnki.cn113664/f.2015.03.001.

[8] Liu, L. J. (2020). Analysis on the problems and countermeasures in the strategic management of enterprises. Modern Marketing (Management Edition), (1), 104. doi: 10.19921/j.cnki.10092994.2020.01.082.

[9] Qu, Z. Q. (2020). Research on the international competition strategy of enterprises in the internet era. Business News, (3), 106-107.

[10] Ren, W. C. (2016). Research on the evolution and development trend of enterprise strategic management theory. China Management Informationization, (12), 91-92.

[11] Shen, Y. (2009). Application of SWOT analysis in the positioning of regional sustainable development [Master's thesis, Xiamen University]. https://kns.cnki.net/KCMS/detail/detail.aspx?dbname=CMFD2009\&filename=2009179637.nh

[12] Tong, Y. F. (2011). Culture leads strategic transformation and innovative development. Chinese Employee Education, (2), 17-19.

[13] Zhang, X. W., \& Zhou, H. M. (2004). The new development and new trend of enterprise strategic management theory. Journal of Hunan Institute of Economic Management, (4), 24-26.

[14] Zuo, H. P., \& Xu, J. X. (2001). The evolution and development of the theory of corporate strategic management. Economic Forum, (19), 31-32. 